\title{
@creative
}

ISSN 2590-9770

The Art of Discrete and Applied Mathematics 1 (2018) \#P1.02

https://doi.org/10.26493/2590-9770.1186.258

(Also available at http://adam-journal.eu)

\section{Hurwitz's regular map $(3,7)$ of genus 7: A polyhedral realization}

\author{
Jürgen Bokowski \\ Department of Mathematics, \\ Technische Universität Darmstadt, \\ Schlossgartenstrasse 7, D-64289 Darmstadt, Germany \\ Michael Cuntz \\ Institut für Algebra, Zahlentheorie und Diskrete Mathematik, \\ Leibniz Universität Hannover, \\ Welfengarten 1, D-30167 Hannover, Germany \\ Dedicated to Prof. Dr. Dr. h.c. Jörg M. Wills \\ on the Occation of his $80^{\text {th }}$ Birthday.
}

Received 19 July 2017, accepted 13 August 2017, published online 10 November 2017

\begin{abstract}
A Hurwitz surface, named after Adolf Hurwitz, is a compact Riemann surface with precisely $84(g-1)$ automorphisms, where $g$ is the genus of the surface. The Hurwitz surface of least genus is the Klein quartic of genus 3. A polyhedral realization without self-intersections of Klein's quartic of genus 3 was found by E. Schulte and J. M. Wills in 1985. For the next possible genus of a Hurwitz surface, i.e., for the genus 7 case with 72 vertices, we provide a polyhedral realization without self-intersections. We also show a topological representation for which we have a corresponding model.
\end{abstract}

Keywords: Hurwitz surface, regular map, polyhedral manifold.

Math. Subj. Class.: $52 B 70$

E-mail addresses: juergen.bokowski@gmail.com (Jürgen Bokowski), cuntz@math.uni-hannover.de (Michael Cuntz)

(a) (i) This work is licensed under http://creativecommons.org/licenses/by/3.0/ 


\section{Introduction}

In Riemann surface theory and hyperbolic geometry, a Hurwitz surface, named after Adolf Hurwitz, is a compact Riemann surface with precisely $84(g-1)$ automorphisms, where $g$ is the genus of the surface. The Hurwitz surface of least genus is the Klein quartic of genus 3 . The next possible genus is 7 with automorphism group $\operatorname{PSL}(2,8)$, which is the simple group of order $84 \times(7-1)=504$; if one includes orientation-reversing isometries, the group is of order 1008 . Our paper is devoted to this genus 7 surface of Adolf Hurwitz from 1893, compare [12], which provides us in modern terminology with a regular map of type $(3,7)_{18}$. We have a closed triangular 2-manifold in which each vertex is incident with seven triangles. The Petrie polygon length is 18 and the automorphism group is flag transitive. In general a regular map is a decomposition of a two dimensional manifold into topological discs, such that every flag can be transformed into any other flag by a symmetry of the decomposition. When we describe a topological disc $d$ via a circular sequence of its vertices $d=\left(v_{1}, v_{2}, \ldots, v_{k}\right)$, a $f$ lag will be in this context a tripel $\left(v_{i},\left(v_{i}, v_{i+1}\right), d\right)$ consisting of a vertex $v_{i}$, an edge $\left(v_{i}, v_{i+1}\right)$, and the disc $d$ itself. For the Hurwitz surface of genus 7, the name Macbeath surface is used as well, although the corresponding article of Macbeath is from 1965, [13].

We find under Wikipedia for regular map: "regular maps are typically defined and studied in three ways: topologically, group-theoretically, and graph-theoretically". However, there are also results in which polyhedral realizations of regular maps have been studied, see e.g. the corresponding articles of Jörg M. Wills and of his co-authors or other colleagues in [2, 3, 4, 5, 6, 7, 8, 16, 17], and [18]. This article is devoted to such a question that was studied by Jörg M. Wills for some time. When only some abstract combinatorial data of a geometric object is given and when we are looking for a corresponding geometric realization or try to prove that no such realization exists, we are facing in general a hard problem that has been called a problem of computational synthetic geometry in [4].

Our main result of this article provides a polyhedral realization of Hurwitz's regular map $(3,7)_{18}$ of genus seven. We also show a topological representation for which we have a corresponding 3D-model. We refer the reader for additional aspects with respect to this paper to the homepage of the second author: http://Www.iazd.uni-hannover. de/cuntz.html.

\subsection{Previous polyhedral realizations of regular maps}

Regular maps generalize on a combinatorial level Platonic solids with their geometric flag transitive automorphism groups. Mani's result [14] asserts that for each combinatorial automorphism of the boundary structure of a convex polyhedron, there does exist a convex polyhedron with a corresponding geometric symmetry. A corresponding result for general regular maps does not hold, the notion hidden symmetries has been used.

The polyhedral realization of Hurwitz's surface of least genus, i.e., a polyhedral realization of Klein's quartic of genus 3, with 24 vertices has been published by E. Schulte and J. M. Wills in [16]. In Figure 1 we have depicted two truncated tetrahedra the vertices of which are the vertices of this symmetric realization.

A first polyhedral realization of a regular map of Walther Dyck $(3,8)_{6}$ with 12 vertices was provided in Antibes in 1987 by Bokowski, see [1] and [2], thus disproving a conjecture of Schulte and Wills that it did not exist. A symmetrical version of this map was found later by Ulrich Brehm, [6]. U. Brehm and U. Leopold have found another polyhedral realization 


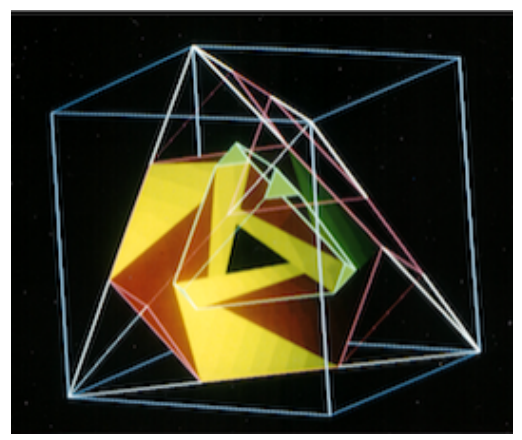

Figure 1: The points of a realization of Hurwitz's regular map of genus 3 by E. Schulte and J. M. Wills are the vertices of two truncated tetrahedra, [16].

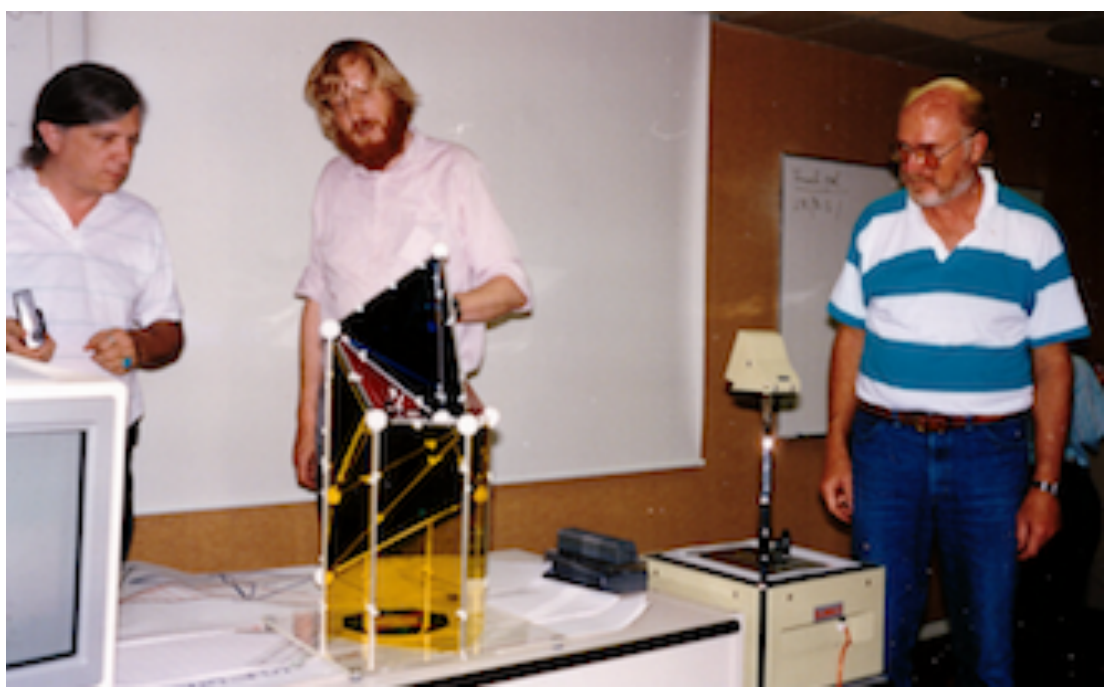

Figure 2: First realization of Dyck's regular map of genus 3 presented by Bokowski (in the middle) in Antibes 1987, [1]. The other two men in the photo are R. Connelly, Cornell University (on the left) and J. M. Wills, University Siegen (on the right). 
of a regular map $(3,10)$ of genus 6 of W. Dyck with 15 vertices, [7]. See also a survey article of U. Brehm and E. Schulte in [8] and the papers cited there.

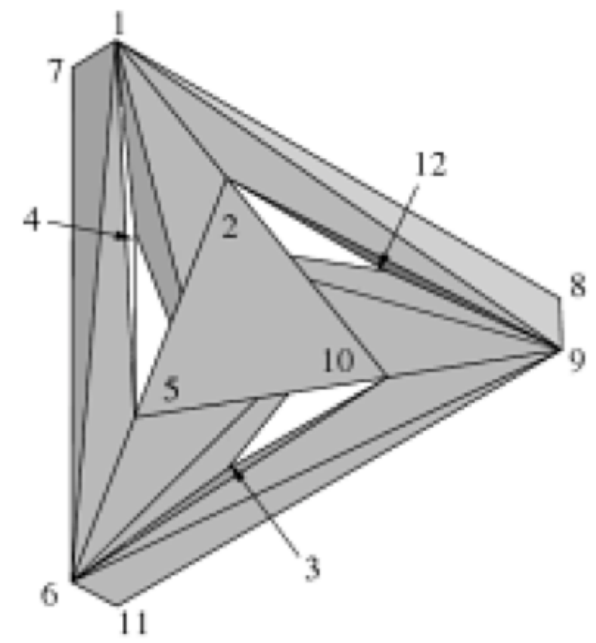

Figure 3: Symmetric realization of Dyck's regular map of genus 3 by U. Brehm [6].

\section{Combinatorial description}

For general descriptions of combinatorial regular maps we refer the reader to [9, 11], and [23]. Hurwitz's regular map of genus 7 consists of the following 168 triangles in Table 1. It has 252 edges and 72 vertices labeled $1, \ldots, 72$.

Compared with previous polyhedral realizations of regular maps we are faced with additional complexity. From the automorphism group of this manifold of order 1008 , we have used a dihedral subgroup of order 14 for sorting the triangles. The cyclic subgroup has the following generator.

$$
\begin{aligned}
(1)(72)(2, \ldots, 8) & (9, \ldots, 15)(16, \ldots, 22)(23, \ldots, 29)(30, \ldots, 36) \\
& (37, \ldots, 43)(44, \ldots, 50)(51, \ldots, 57)(58, \ldots, 64)(65, \ldots, 71)
\end{aligned}
$$

When we assume the vertices from 2 to 71 to form ten regular seven-gons in horizontal equidistant planes with heights sorted according to labels belonging to the same orbit of the cyclic group, we have an additional up-side-down symmetry that maps vertex 1 to vertex 72 which is an automorphism of the map.

The combinatorial description of a complete list of small regular maps has been given in [10], an even more extended list is available from the first author of the same article.

\section{Topological visualization}

The study of topological visualizations of regular maps has recently been done by J. J. van Wijk, [21, 22], by C. H. Séquin, [20], and by Razafindrazaka and Polthier, [15]. From J. J. van Wijk we have a nice topological visualization as a computer film of our Hur- 
Table 1: Triangles of Hurwitz's surface of genus seven.

$\begin{array}{llllll}(01,02,03), & (01,03,04), & (01,04,05), & (01,05,06), & (01,06,07), & (01,07,08), \\ (01,08,02), & (02,08,09), & (02,10,03), & (03,11,04), & (12,05,04), & (13,06,05), \\ (14,07,06), & (08,07,15), & (02,09,23), & (03,10,24), & (11,25,04), & (12,26,05), \\ (13,27,06), & (14,28,07), & (08,15,29), & (02,16,10), & (03,17,11), & (12,04,18), \\ (13,05,19), & (20,14,06), & (21,15,07), & (22,09,08), & (02,23,16), & (03,24,17), \\ (04,25,18), & (19,05,26), & (20,06,27), & (21,07,28), & (22,08,29), & (22,51,09), \\ (16,52,10), & (11,17,53), & (12,18,54), & (55,13,19), & (56,14,20), & (21,57,15), \\ (46,23,09), & (47,24,10), & (48,25,11), & (49,26,12), & (50,27,13), & (44,28,14), \\ (45,29,15), & (46,09,32), & (47,10,33), & (48,11,34), & (49,12,35), & (50,13,36), \\ (44,14,30), & (45,15,31), & (32,09,51), & (10,52,33), & (11,53,34), & (12,54,35), \\ (55,36,13), & (30,14,56), & (31,15,57), & (36,16,23), & (30,17,24), & (31,18,25), \\ (32,19,26), & (20,27,33), & (21,28,34), & (22,29,35), & (36,42,16), & (30,43,17), \\ (31,37,18), & (38,19,32), & (39,20,33), & (21,34,40), & (22,35,41), & (58,16,42), \\ (59,17,43), & (37,60,18), & (38,61,19), & (39,62,20), & (21,40,63), & (64,22,41), \\ (58,52,16), & (59,53,17), & (54,18,60), & (55,19,61), & (56,20,62), & (21,63,57), \\ (64,51,22), & (36,23,37), & (30,24,38), & (39,31,25), & (40,32,26), & (41,33,27), \\ (34,28,42), & (35,29,43), & (37,23,60), & (38,24,61), & (39,25,62), & (40,26,63), \\ (64,41,27), & (58,42,28), & (59,43,29), & (46,60,23), & (47,61,24), & (48,62,25), \\ (49,63,26), & (50,64,27), & (44,58,28), & (45,59,29), & (44,30,38), & (45,31,39), \\ (46,32,40), & (47,33,41), & (48,34,42), & (49,35,43), & (50,36,37), & (30,56,43), \\ (31,57,37), & (38,32,51), & (39,33,52), & (40,34,53), & (54,41,35), & (55,42,36), \\ (50,37,57), & (44,38,51), & (45,39,52), & (46,40,53), & (47,41,54), & (48,42,55), \\ (49,43,56), & (4451,65), & (45,52,66), & (46,53,67), & (47,54,68), & (48,55,69), \\ (49,56,70), & (50,57,71), & (44,65,58), & (45,66,59), & (46,67,60), & (47,68,61), \\ (48,69,62), & (49,70,63), & (50,71,64), & (64,65,51), & (58,66,52), & (67,53,59), \\ (68,54,60), & (55,61,69), & (56,62,70), & (63,71,57), & (58,65,66), & (67,59,66), \\ (67,68,60), & (68,69,61), & (69,70,62), & (63,70,71), & (64,71,65), & (66,65,72), \\ (67,66,72), & (67,72,68), & (68,72,69), & (69,72,70), & (71,70,72), & (65,71,72),\end{array}$


witz surface of genus 7 in [22]. We show corresponding pictures with a dihedral symmetry $D_{7}$ of order 14 in Figure 4 and Figure 5.

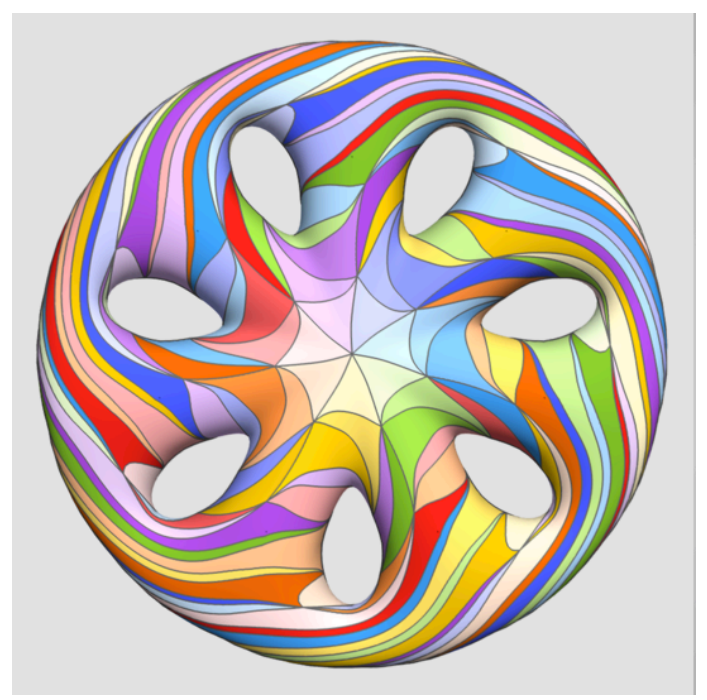

Figure 4: Topological visualization of Hurwitz's regular map $(3,7)$ of genus 7 of J. J. van Wijk, [21].
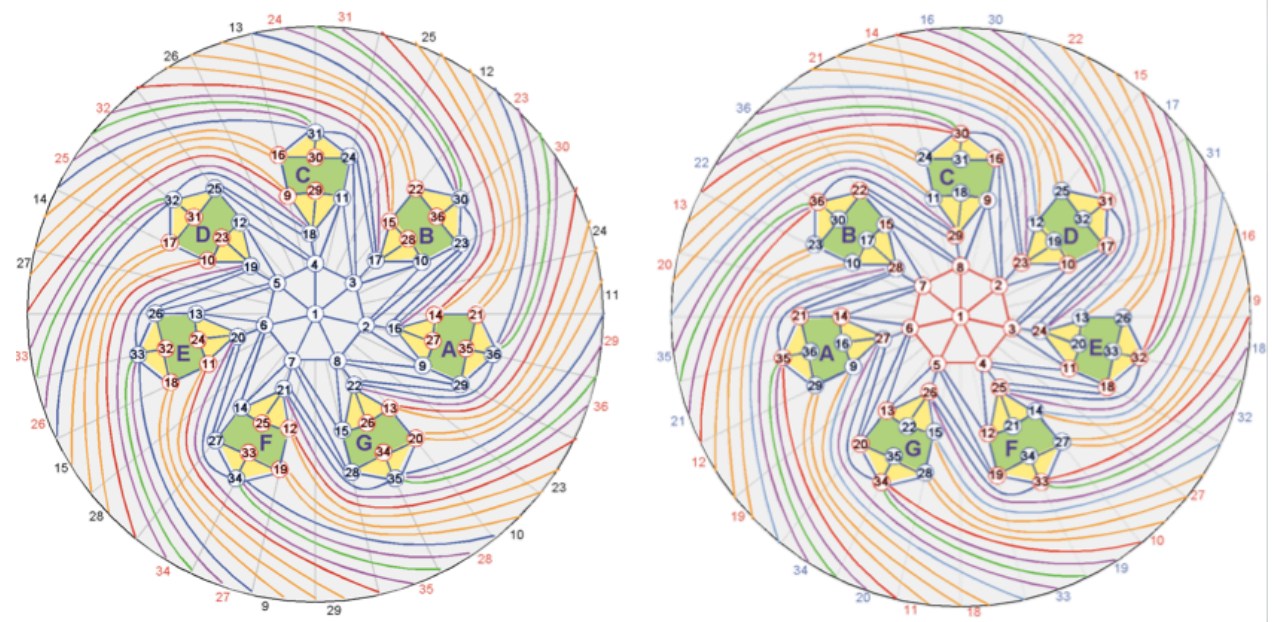

Figure 5: Topological visualization of Hurwitz's regular map $(3,7)$ of genus 7 of C. H. Séquin, [20], see also [19].

Unfortunately, the method of Razafindrazaka and Polthier did not work in the case of Hurwitz's surface of genus 7 to provide an additional topological visualization. However, we have an additional different topological visualization as a 3D-Model that was helpful during our investigation for finding a polyhedral realization, see Figure 6. 
This model of Figure 6 shows seven six-gons (marked by white connections inside the outer torus) around the axis that is fixed under the dihedral symmetry.

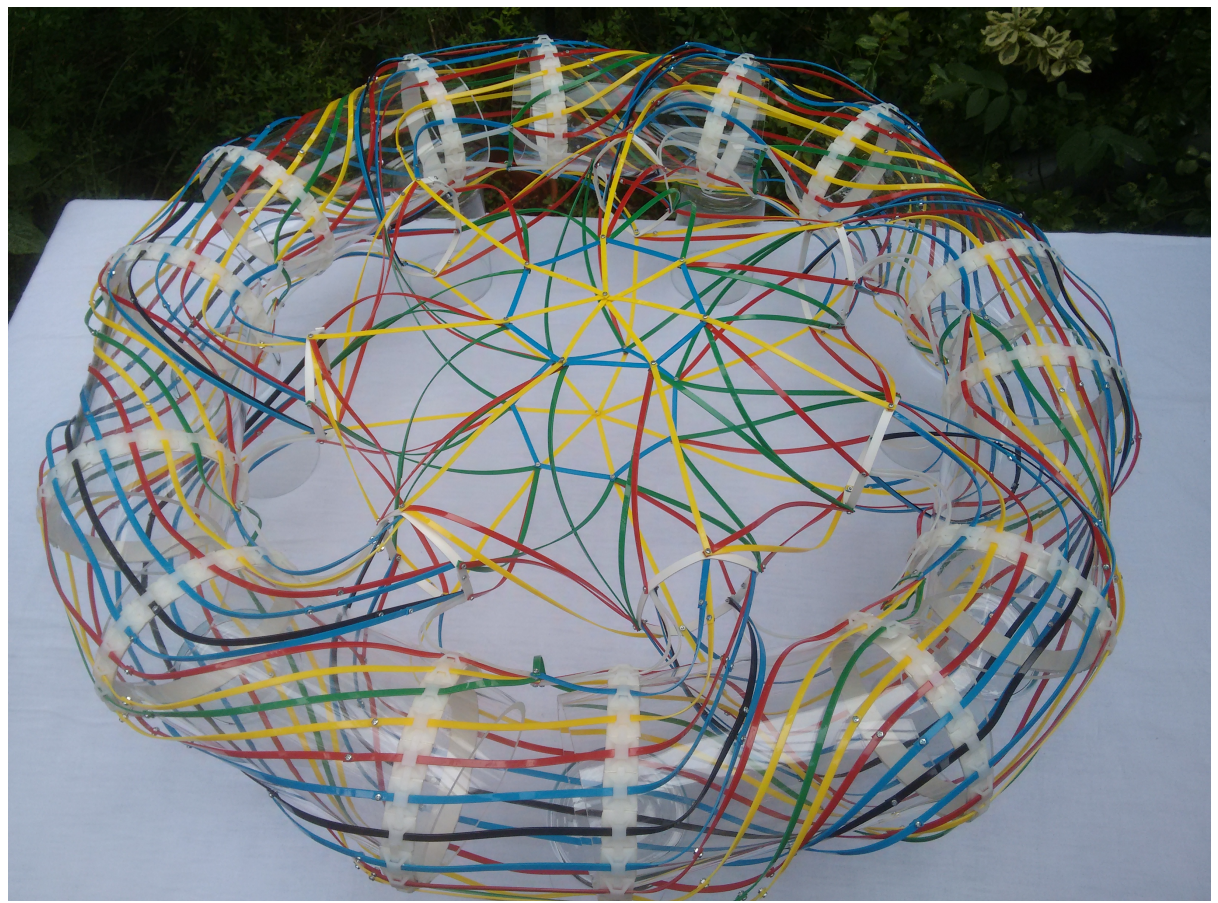

Figure 6: Topological visualization of Hurwitz's regular map $(3,7)$ of genus 7 as a 3Dmodel. (This model was presented at the Jörgshop at the Technical University Berlin in June 2017.)

When we cut the model along those six-gons, we see that we can split the surface in two parts having 84 triangles each. On the one hand we obtain a topological torus with these seven six-gons as holes and on the other hand we have a topological 2-sphere with these seven holes.

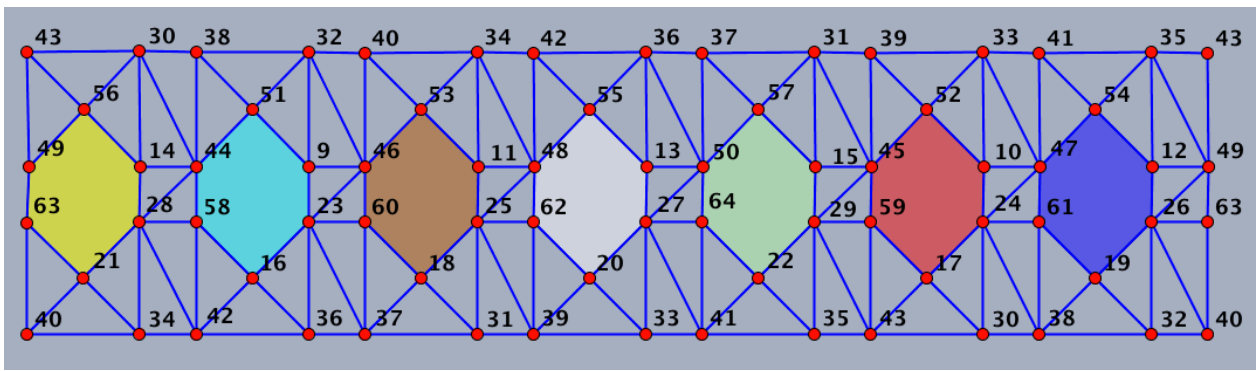

Figure 7: Triangles of the torus with seven holes each bounded by a polygon of length 6 .

In Figure 7 we have depicted the combinatorial torus structure and in Figure 8 we see the corresponding 84 triangles of the sphere. Whereas both of these parts of the Hur- 
witz surface of genus 7 can easily be represented with planar triangles and without selfintersections, we see that the cyclic sequences of the holes in both cases do not coincide. However, they have to match. This is a clear indication that we probably cannot hope for a corresponding symmetric realization of order 7 .

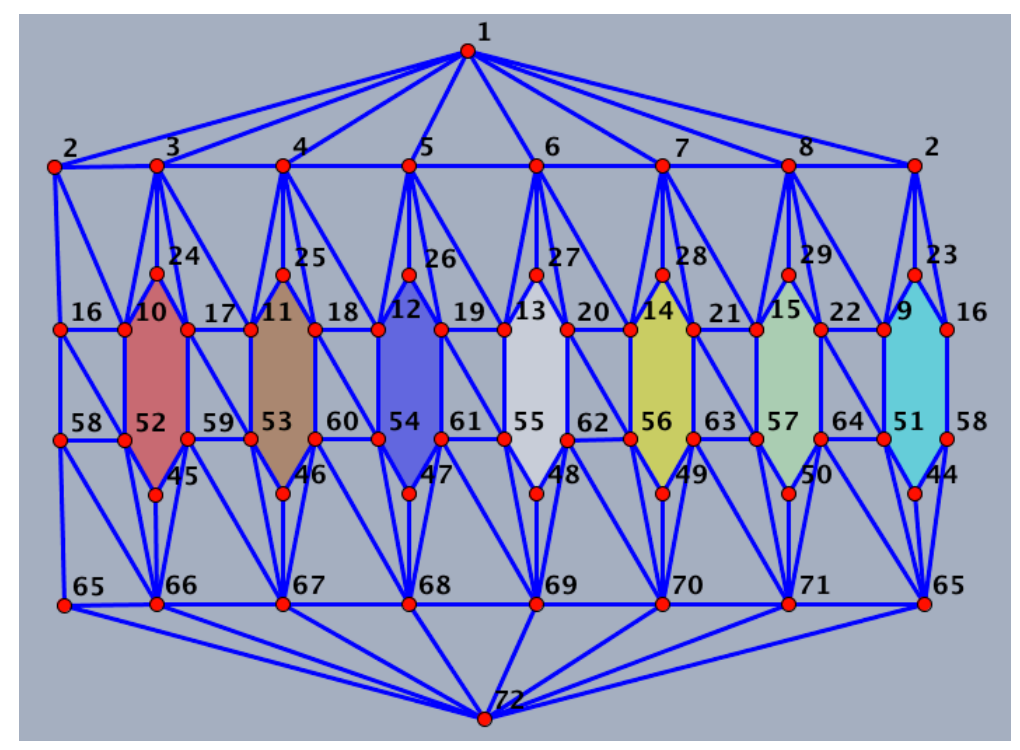

Figure 8: Triangles of the sphere with seven holes each bounded by a polygon of length 6 .

\section{Polyhedral realization}

\subsection{An algorithm}

We use the following simple algorithm to obtain realizations within a few minutes (depending on the choice of distances and parameters):

1. Choose randomly a set of 72 distinct points $P=\left\{P_{1}, \ldots, P_{72}\right\} \subseteq \mathbb{Q}^{3}$ with rational coordinates.

2. Count the number $w_{0}$ of pairs of labels of a triangle and labels of an edge of a triangle in Table 1 for which the corresponding points in $P$ produce an intersection of a triangle and an edge.

3. Remember the points involved in these $w_{0}$ intersections in a set $I$.

4. While $w_{0}>0$, do:

(a) Move a randomly chosen point of $I$ into a random direction in such a way that it does not go too far away and not too close to the other points.

(b) As above, count the number $w$ of intersections and remember the points involved in intersections in a new set $I$.

(c) If $w>w_{0}$ then move the point back to its place, else: $w_{0} \leftarrow w$.

5. Output the solution. 
An implementation in $\mathrm{C}$ produces for example the solution displayed in Table 2. To be completely sure that this output is correct one may check it using the code in Figure 9 or Figure 10.

Table 2: Coordinates of a polyhedral realization without self-intersections of Hurwitz's surface of genus seven.

$\begin{array}{rrrrrrrrrrrr}\text { no. } & \mathrm{x} & \mathrm{y} & \mathrm{z} & \text { no. } & \mathrm{x} & \mathrm{y} & \mathrm{z} & \text { no. } & \mathrm{x} & \mathrm{y} & \mathrm{z} \\ 1 & 430 & -270 & -1000 & 2 & 959 & -237 & -213 & 3 & 434 & -984 & -70 \\ 4 & -418 & -861 & -677 & 5 & -988 & 98 & -665 & 6 & -272 & -139 & -814 \\ 7 & 299 & 577 & -988 & 8 & 999 & 399 & -854 & 9 & 981 & 727 & -246 \\ 10 & 475 & -498 & 408 & 11 & 361 & -806 & 840 & 12 & -509 & 115 & 609 \\ 13 & -541, & -105 & 26 & 14 & -299 & 434 & -801 & 15 & 456 & -230 & -780 \\ 16 & 819 & 353 & 803 & 17 & 841 & -663 & 868 & 18 & -941 & 982 & 856 \\ 19 & -928 & 694 & -73 & 20 & 21 & -294 & 158 & 21 & -132 & 450 & -319 \\ 22 & 526 & 305 & -430 & 23 & 782 & -550 & 996 & 24 & 172 & -288 & 93 \\ 25 & -859 & -989 & 528 & 26 & -679 & 983 & 697 & 27 & -95 & -239 & -217 \\ 28 & 764 & 665 & 653 & 29 & 563 & 490 & 169 & 30 & -872 & 507 & -510 \\ 31 & -413 & -817 & -561 & 32 & 136 & 921 & 30 & 33 & 432 & -176 & -157 \\ 34 & 522 & 778 & 359 & 35 & 489 & -85 & 120 & 36 & -470 & 84 & 709 \\ 37 & -520 & -823 & 679 & 38 & -383 & 876 & -325 & 39 & 365 & -758 & -25 \\ 40 & 114 & 900 & 838 & 41 & 240 & 176 & -191 & 42 & 234 & 26 & 700 \\ 43 & 4 & -150 & 345 & 44 & 261 & 843 & -15 & 45 & 850 & 19 & -196 \\ 46 & 902 & 679 & 797 & 47 & 17 & 36 & 114 & 48 & -331 & -763 & 720 \\ 49 & -523 & 632 & 368 & 50 & -254 & -694 & -243 & 51 & 367 & 659 & -796 \\ 52 & 791 & -11 & 367 & 53 & 194 & 442 & 411 & 54 & 34 & 376 & 304 \\ 55 & -132 & -413 & 773 & 56 & 103 & -743 & 654 & 57 & -240 & -160 & -832 \\ 58 & 940 & 632 & 175 & 59 & 567 & -43 & 515 & 60 & 224 & 233 & 981 \\ 61 & -254 & 268 & 182 & 62 & -271 & -721 & 265 & 63 & -60 & 540 & 192 \\ 64 & 280 & -119 & -630 & 65 & 644 & 565 & -266 & 66 & 516 & 538 & 265 \\ 67 & -117 & 524 & 443 & 68 & 210 & 227 & -110 & 69 & -275 & -204 & 444 \\ 70 & -157 & 44 & 359 & 71 & 199 & 402 & -282 & 72 & 90 & 510 & 140\end{array}$

\subsection{Explanations}

It is already very difficult to describe the geometric shape of any of the two parts of the Hurwitz surface of genus 7 that we have described in the last section. The Blender software is a powerful tool for 3D objects. In Figure 11, Figure 12, Figure 13, and Figure 14 you see some pictures of our realization. The reader can get a better understanding by using our corresponding Blender files for rotating the objects. Please write an e-mail to the authors. Glueing properly both parts along their boundaries leads to our polyhedral realization without self-intersections.

How did we check that the polyhedron has no self-intersections? We first confirmed that all vertices are in general position. This is equivalent to the fact that all determinants of any 4 points (by using homogeneous coordinates) are non-zero. Afterwards we have checked all pairs (edge, triangle) for intersections. Edges that have a vertex in common with a triangle cause no problem, because the points are in general position.

The other cases (edge, triangle) depend on the signs of the five determinants obtained from the five 4 -element subsets of the set formed by the vertices of the edge and the trian- 
gle (again using homogeneous coordinates). When the two vertices of an edge lie on the same side of the plane determined by the triangle, we have no intersection. Otherwise we pick a vertex of the edge as the apex of a cone generated by the triangle. Precisely, when all three planes determined by the faces of this cone have the other vertex of the edge on the same side as the remaining vertex of the triangle, we have an intersection. In other words the other vertex of the edge lies within the convex cone, however beyond the triangle seen from the apex. We have double checked this result with two different programming methods, Haskell and Magma. When using exploded views, corresponding films, a symmetric realization, or even a geometric model, the reader might gain additional insight. Our attempts to find a symmetric realization were not successful. For a cyclic symmetry of order 7 we have even seen an argument that tells us how unlikely the existence of such a realization might be.

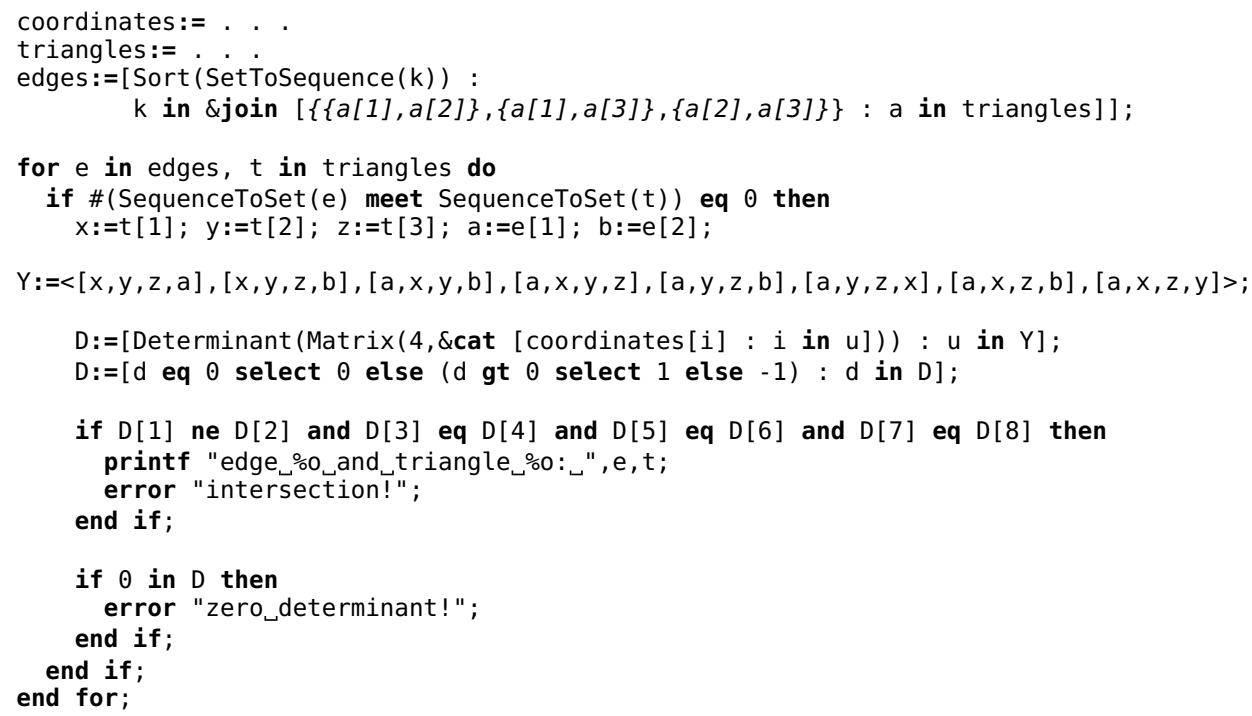

Figure 9: Magma code. 


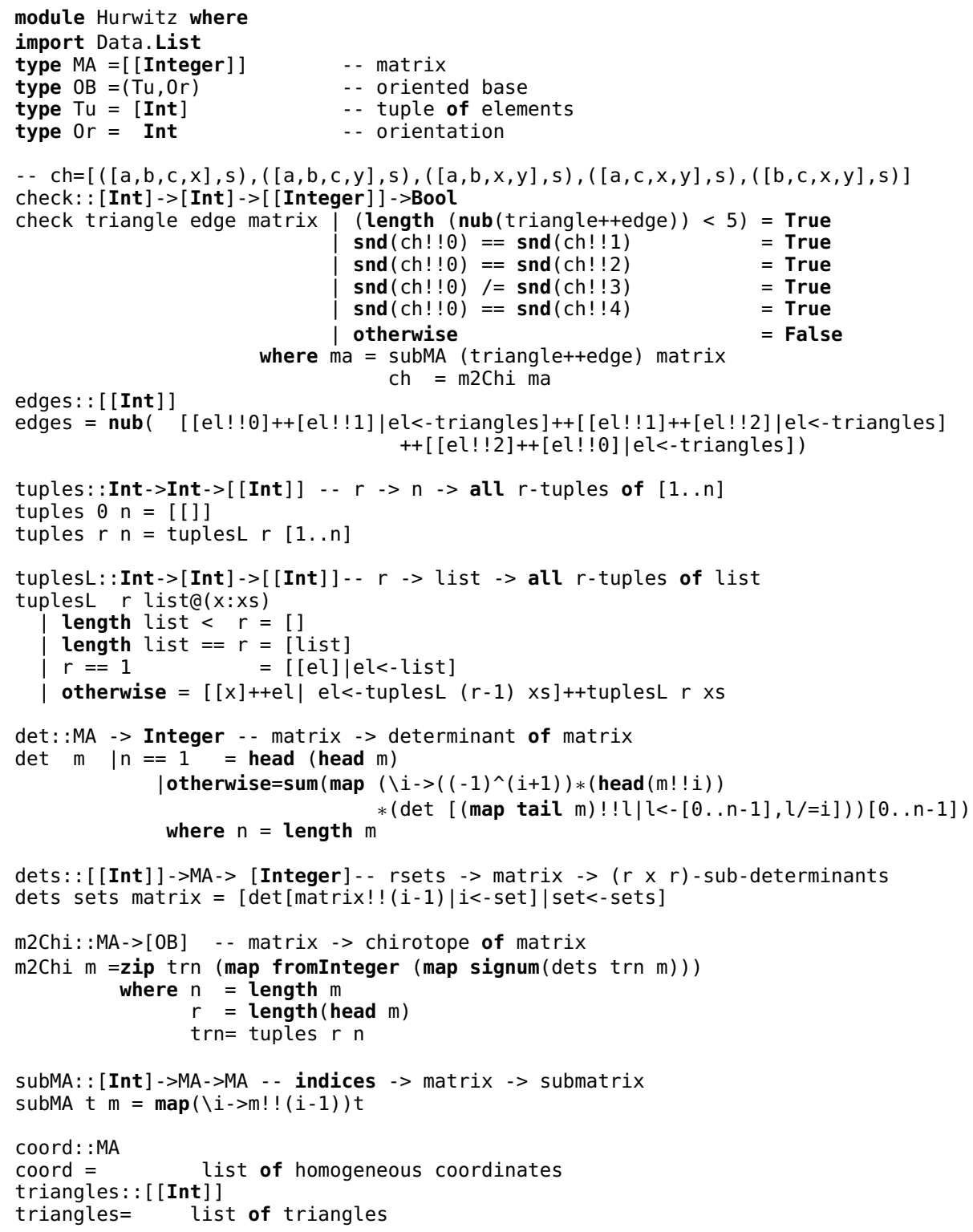

Figure 10: Haskell code with some explanations. 


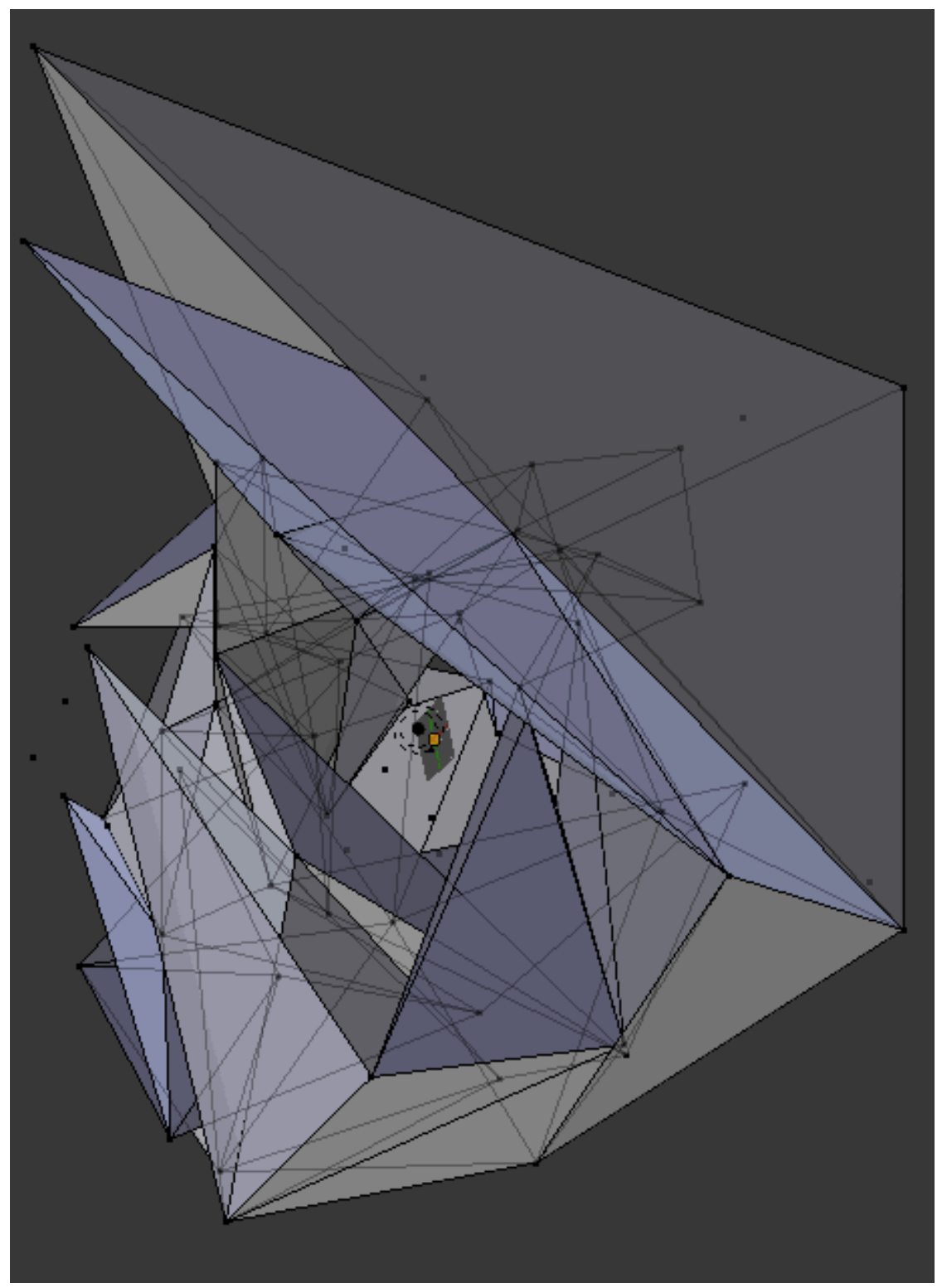

Figure 11: Polyhedral realization of the sphere with seven holes each bounded by a polygon of length 6 . 


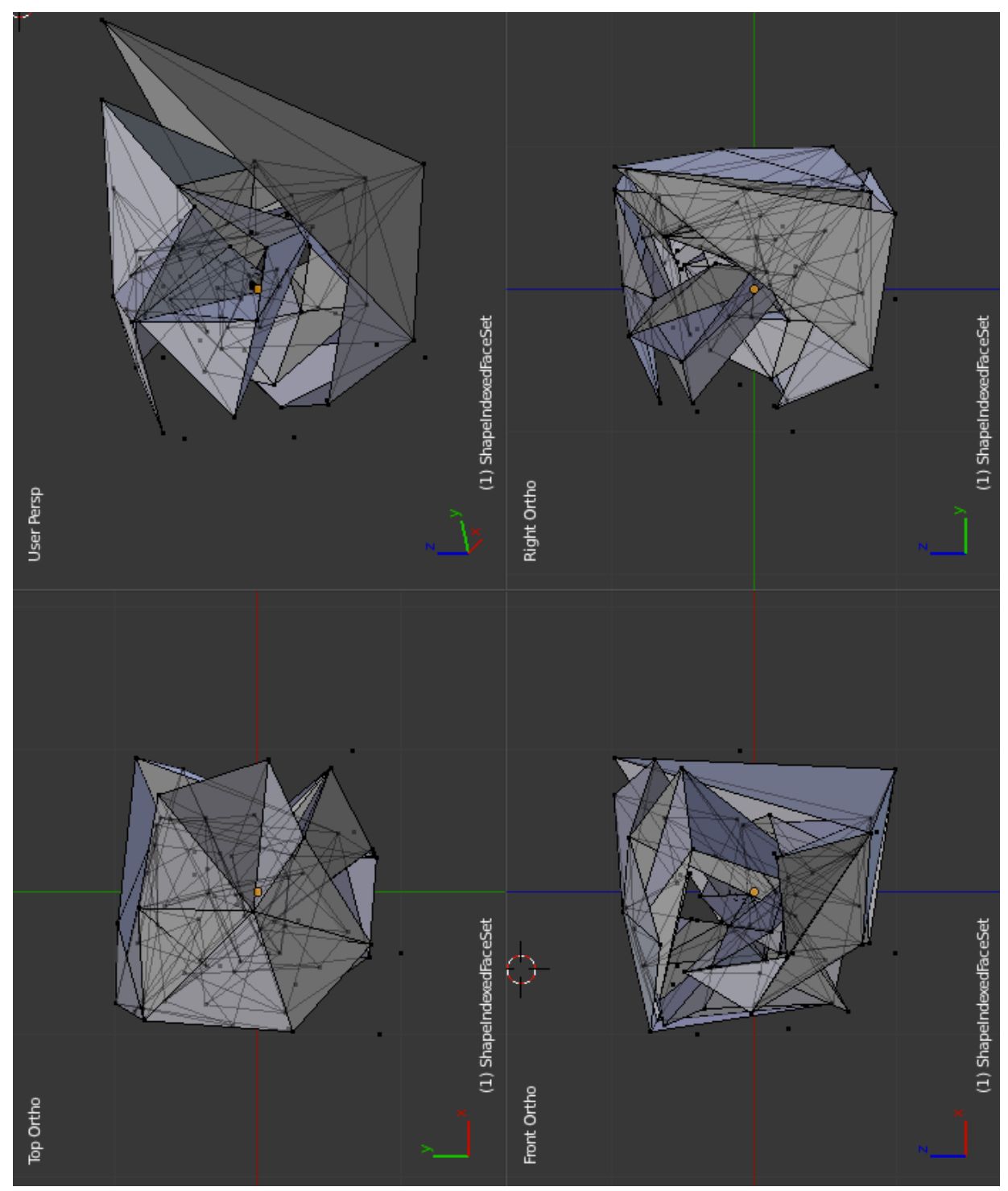

Figure 12: Polyhedral realization of the torus with seven holes each bounded by a polygon of length 6. Three orthogonal projections and a perspective view. Even half of the complete polyhedral realization is difficult to understand. 


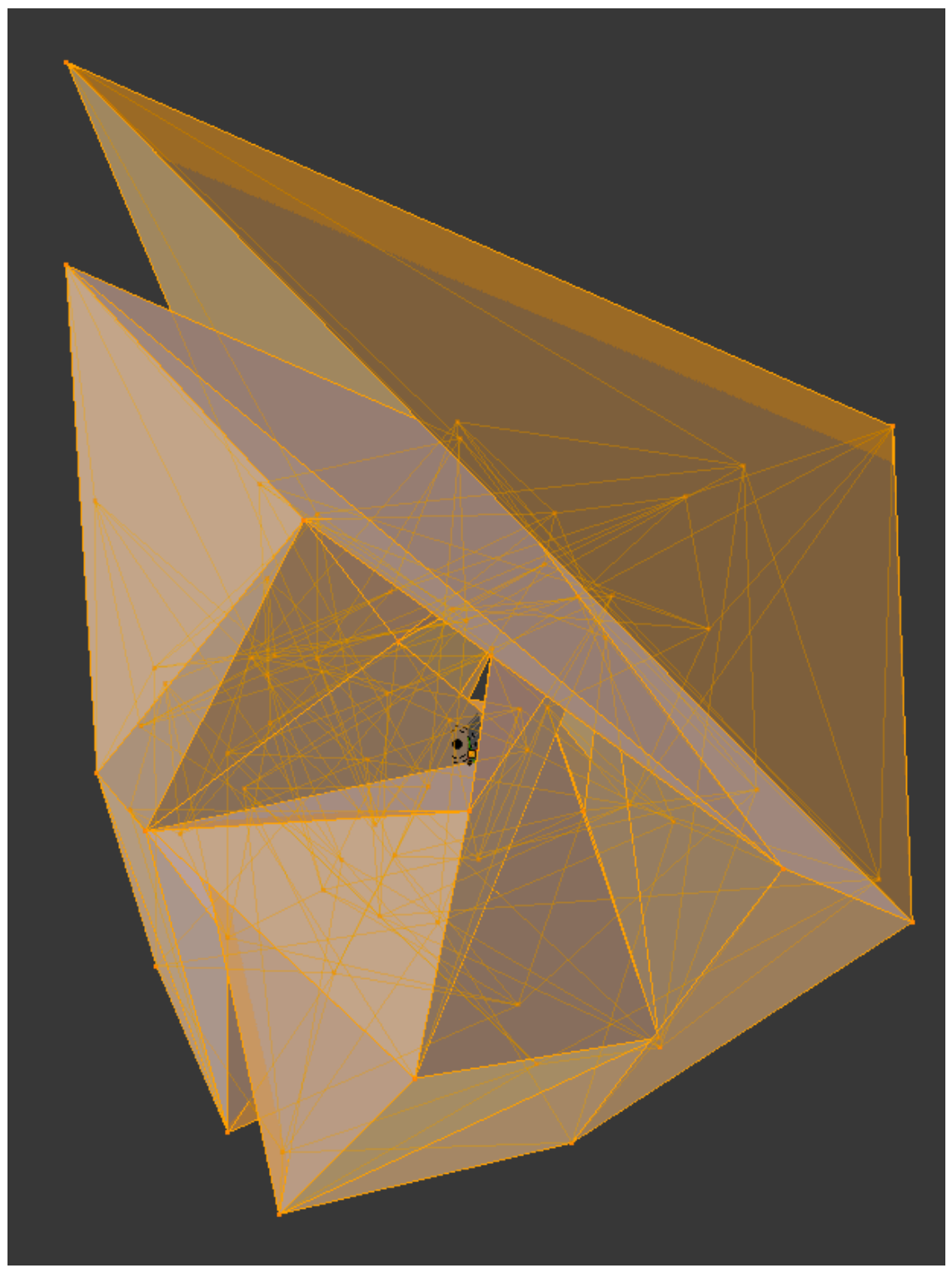

Figure 13: Polyhedral realization Hurwitz's surface of genus 7. 


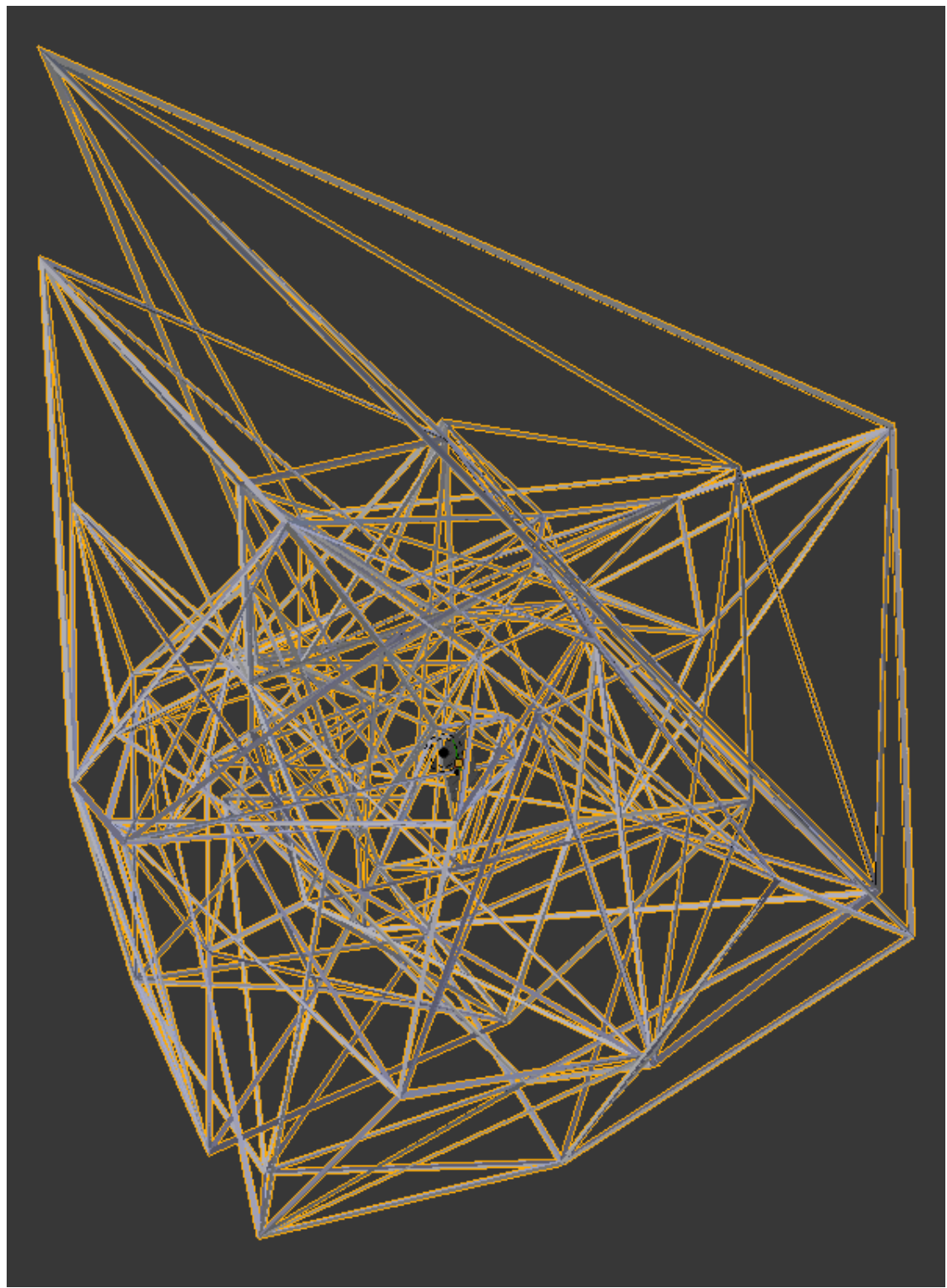

Figure 14: Polyhedral realization Hurwitz's surface of genus 7, complete wireframe. 


\section{References}

[1] J. Bokowski, Aspects of computational synthetic geometry II: Combinatorial complexes and their geometric realization - An algorithmic approach, in: H. Crapo (ed.), Computer-Aided Geometric Reasoning, Institut National de Recherche en Informatique et en Automatique (INRIA), Antibes, France, 1987 pp. 87-125, proceedings of the INRIA Workshop (Sophia Antipolis, 1987).

[2] J. Bokowski, A geometric realization without self-intersections does exist for Dyck's regular map, Discrete Comput. Geom. 4 (1989), 583-589, doi:10.1007/bf02187748.

[3] J. Bokowski, On the geometric flat embedding of abstract complexes with symmetries, in: K. H. Hofmann and R. Wille (eds.), Symmetry of Discrete Mathematical Structures and Their Symmetry Groups, Heldermann, Berlin, volume 15 of Research and Exposition in Mathematics, 1991 pp. 1-48, a collection of essays.

[4] J. Bokowski and B. Sturmfels, Computational Synthetic Geometry, volume 1355 of Lecture Notes in Mathematics, Springer-Verlag, Berlin, 1989, doi:10.1007/bfb0089253.

[5] J. Bokowski and J. M. Wills, Regular polyhedra with hidden symmetries, Math. Intell. 10 (1988), 27-32, doi:10.1007/bf03023848.

[6] U. Brehm, Maximally symmetric polyhedral realizations of Dyck's regular map, Mathematika 34 (1987), 229-236, doi:10.1112/s0025579300013474.

[7] U. Brehm and U. Leopold, A symmetric combinatorially regular polyhedron of genus 6 with 15 vertices, in preparation.

[8] U. Brehm and E. Schulte, Polyhedral maps, in: J. E. Goodman and J. O'Rourke (eds.), Handbook of Discrete and Computational Geometry, CRC Press, Boca Raton, Florida, CRC Press Series on Discrete Mathematics and its Applications, 1997 pp. 345-358.

[9] M. Conder, Orientable regular maps of genus 2 to 101, 2006, http://www.math. auckland.ac.nz/ conder/OrientableRegularMaps101.txt.

[10] M. Conder and P. Dobcsányi, Determination of all regular maps of small genus, J. Comb. Theory Ser. B 81 (2001), 224-242, doi:10.1006/jctb.2000.2008.

[11] H. S. M. Coxeter and W. O. J. Moser, Generators and Relations for Discrete Groups, volume 14 of Ergebnisse der Mathematik und ihrer Grenzgebiete, Springer-Verlag, Berlin, 4th edition, 1980, doi:10.1007/978-3-662-21943-0.

[12] A. Hurwitz, Ueber algebraische Gebilde mit eindeutigen Transformationen in sich, Math. Ann. 41 (1893), 403-442, doi:10.1007/bf01443420.

[13] A. M. Macbeath, On a curve of genus 7, Proc. London Math. Soc. 15 (1965), 527-542, doi: 10.1112/plms/s3-15.1.527.

[14] P. Mani, Automorphismen von polyedrischen Graphen, Math. Ann. 192 (1971), 279-303, doi: $10.1007 / \mathrm{bf02075357.}$

[15] F. Razafindrazaka and K. Polthier, Regular surfaces and regular maps, in: G. Greenfield, G. Hart and R. Sarhangi (eds.), Proceedings of Bridges 2014: Mathematics, Music, Art, Architecture, Culture (Seoul, Korea, 2014), Tessellations Publishing, Phoenix, Arizona, 2014 pp. 225-234, http://archive.bridgesmathart.org/ 2014/bridges2014-225.html.

[16] E. Schulte and J. M. Wills, A polyhedral realization of Felix Klein's map $\{3,7\}_{8}$ on a Riemann surface of genus 3, J. London Math. Soc. 32 (1985), 539-547, doi:10.1112/jlms/s2-32.3.539.

[17] E. Schulte and J. M. Wills, Combinatorially regular polyhedra in three-space, in: K. H. Hofmann and R. Wille (eds.), Symmetry of Discrete Mathematical Structures and Their Symmetry 
Groups, Heldermann, Berlin, volume 15 of Research and Exposition in Mathematics, 1991 pp. 49-88, a collection of essays.

[18] E. Schulte and J. M. Wills, Convex-faced combinatorially regular polyhedra of small genus, Symmetry 4 (2012), 1-14, doi:10.3390/sym4010001.

[19] C. H. Séquin, My search for symmetrical embeddings of regular maps, in: G. W. Hart and R. Sarhangi (eds.), Proceedings of Bridges 2010: Mathematics, Music, Art, Architecture, Culture (Pécs, Hungary, 2010), Tessellations Publishing, Phoenix, Arizona, 2010 pp. 85-94, http://archive.bridgesmathart.org/2010/bridges2010-85.html.

[20] C. H. Séquin, private communication to J. J. van Wijk, 2010.

[21] J. J. van Wijk, Symmetric tiling of closed surfaces: visualization of regular maps, ACM Trans. Graph. 28 (2009), 49:1-49:12, doi:10.1145/1531326.1531355.

[22] J. J. van Wijk, Visualization of regular maps: the chase continues, IEEE Trans. Vis. Comput. Graphics 20 (2014), 2614-2623, doi:10.1109/tvcg.2014.2352952.

[23] A. Vince, Maps, in: J. L. Gross and J. Yellen (eds.), Handbook of Graph Theory, CRC Press, Boca Raton, Florida, Discrete Mathematics and its Applications, 2004. 